\title{
The quest for novel modes of excitation in exotic nuclei
}

\author{
N. Paar \\ Physics Department, Faculty of Science, University of Zagreb, Bijenička 32, 10000 \\ Zagreb, Croatia \\ E-mail: npaar@phy.hr
}

\begin{abstract}
This article provides an insight into several open problems in the quest for novel modes of excitation in nuclei with isospin asymmetry, deformation and finite temperature characteristic in stellar environment. Major unsolved problems include the nature of pygmy dipole resonances, the quest for various multipole and spinisospin excitations both in neutron-rich and proton drip-line nuclei mainly driven by loosely bound nucleons, excitations in unstable deformed nuclei and evolution of their properties with the shape phase transition. Exotic modes of excitation in nuclei at finite temperatures characteristic for supernova evolution present open problems with possible impact in modeling astrophysically relevant weak interaction rates. All these issues challenge self-consistent many body theory frameworks at the frontiers of ongoing research, including nuclear energy density functionals, both phenomenological and constrained by the strong interaction physics of QCD, models based on lowmomentum two-nucleon interaction $V_{l o w-k}$ and correlated realistic nucleon-nucleon interaction $V_{U C O M}$, supplemented by three-body force, as well as two-nucleon and three-nucleon interactions derived from the chiral effective field theory. Joined theoretical and experimental efforts, including research with radioactive isotope beams, are needed to provide insight into dynamical properties of nuclei away from the valley of stability, involving the interplay of isospin asymmetry, deformation and finite temperature.
\end{abstract}

PACS numbers: 21.10.Gv, 21.30.Fe, 21.60.Jz, 24.30.Cz,13.75.Cs 


\section{Introduction}

Nuclei away from the valley of stability are characterized by unique structure properties including weak binding of outermost nucleons, coupling between bound states and the particle continuum, diffuse neutron density distributions, formation of neutron skin and halo structures. Due to these properties, the structure of multipole response in unstable nuclei can be modified and novel exotic modes of excitation may appear. In particular, the dipole response of neutron-rich nuclei is characterized by the fragmentation of the strength distribution and its spreading into the low-energy region, and by the mixing of isoscalar and isovector components [1]. While in light nuclei the onset of dipole strength in the low-energy region is caused by non-resonant independent single-particle excitations of the loosely bound neutrons, several theoretical analyses have predicted the existence of the pygmy dipole resonance (PDR) in medium-mass and heavy nuclei, i.e. the resonant oscillation of the weakly-bound neutron skin against the isospin saturated proton neutron core. Although the properties of the PDR have been studied with numerous many body methods and effective nuclear interactions, there is a considerable number of open questions about its dynamics that are at present time under discussion.

The richness of the structure properties and numerous challenging results about the nature of PDR open perspectives to develop a whole new area of research on other exotic modes of excitation in weakly bound nuclei. One could expect, at least in principle, that the structure properties of exotic nuclei could result in novel modes of excitation of various multipolarities, in charge-exchange channel and nuclei with deformation, as well as in nuclei at finite temperatures characteristic for the stellar environment and supernova evolution.

Exotic modes of excitation probe several aspects of the underlying structure properties and effective nuclear interactions, including the in-medium modification of the nucleon-nucleon interaction, and the interplay between different degrees of freedom in dissipative processes that determine the damping mechanism. These modes could also provide sensitive test of novel microscopic theory frameworks based on nuclear energy density functional [2, 3, 4, 5, 6, 7, 8, 9] or low-momentum two-nucleon (NN) interactions, $V_{\text {low-k }}$ based on renormalisation group transformations [10] or $V_{U C O M}$ obtained by the unitary correlation operator method [11. $V_{l o w-k}$ has also been supplemented with a three-body force (NNN) [12]. New developments in connecting the QCD with low-energy nuclear physics through chiral effective field theory $(\chi \mathrm{EFT})$, which resulted in consistent derivation of NN and NNN forces, could also provide challenging theory framework to probe the structure properties of exotic modes [13]. Progress in theoretical predictions of exotic modes has also initiated a number of experimental studies on low-energy multipole response, including for instance scattering of photons, protons, and alpha particles on nuclei, as well as excitations involving radioactive isotope beams (e.g. Refs. [14] -[27]).

In the following sections an overview is given on several challenging research topics on exotic modes of excitation in nuclei, that are not understood at present time, and could be addressed in the future by theory frameworks either based on modern 
energy density functionals or low-momentum NN interactions supplemented by NNN forces. All these research topics necessitate strong support from the experiment, in order to validate theoretical methods, findings, and implementation of various effective interactions in modeling the nuclear many-body problem under conditions of pronounced isospin asymmetry, deformation, and finite temperature.

\section{Self-consistent theory of excitations in exotic nuclei}

One of the central aims in nuclear physics is the construction of effective interactions suitable for self-consistent solving the nuclear many-body problem, either within the nuclear energy density functional theory or models based on realistic NN and NNN interactions. In the former case, the parameters of effective interactions are usually constrained at the level of the self-consistent mean field model by selection of data on the ground state properties of atomic nuclei and nuclear matter. The most prominent selfconsistent mean-field models for describing nuclear structure and low-energy dynamics are based on Skyrme energy functional, finite range Gogny interaction, and relativistic energy density functional [2]. More recently, these frameworks also provided insight into various aspects of nuclear structure in unstable nuclei, including exotic modes of excitation in neutron-rich nuclei, e.g. pygmy dipole resonance [1], di-neutron vibration mode [28], low-energy quadrupole response [29, 30], etc. The self-consistent RPA with Gogny interaction, using the Gaussian expansion method which includes coupling to the continuum enables studies of low-energy multipole excitations in drip-line nuclei [31.

Excitations involving weakly bound nucleons represent very fine structure phenomena, and could be used as sensitive benchmarks in construction of novel effective nuclear interactions and many body methods. For a microscopic description of transition spectra spreading widths, of particular importance are the effects due to coupling to complex configurations. In a recent formulation of relativistic quasiparticle time blocking approximation, coupling of two-quasiparticle excitations to collective vibrations is included within a fully consistent calculation scheme based on covariant energy density functional theory [32, 33]. Another approach which takes into account complex configurations, second RPA (SRPA), is based on extension of the RPA which, in addition to $p h$ configurations, also includes $2 p 2 h$ excitations and residual interaction terms that couple $p h$ and $2 p 2 h$ configurations, as well as $2 p 2 h$ configurations among themselves. However, due to numerical difficulties, the SRPA equations have usually been solved by reducing to Tamm-Dancoff approach, the residual interaction terms that couple $2 p 2 h$ with $2 p 2 h$ configurations have been neglected, severe restrictions on the configuration space have been imposed, and there have been no consistency between the ground state description and residual SRPA interaction terms. Only very recently, self-consistent SRPA have been established, based on correlated Argonne V18 [34] and Skyrme effective interactions [35], and employed in the initial studies of giant resonances and low-lying $0^{+}$ and $2^{+}$states. Future developments and studies based on SRPA could provide crucial information about excitations in unstable nuclei and their spreading widths. 
At present time exotic modes of excitation are still out of reach for theory frameworks starting from the strong interaction physics of QCD. One promising approach could be the nuclear energy density functional theory (NEDF), based on chiral dynamics and the symmetry breaking pattern of low-energy QCD [7], and recently extended to the description of collective nuclear excitations [36]. The proton-neutron random phase approximation, based on relativistic point-coupling Lagrangian, has been applied to investigate the role of chiral pion-nucleon dynamics in excitation modes involving spin and isospin degrees of freedom, e.g. isobaric analog states and GamowTeller resonances. The NEDF framework could be further extended for studies of other excitation modes of interest. Nuclear potentials have also been constructed in another approach based on effective field theories, that respect the symmetry pattern of QCD and produce observables in a systematic and controlled expansion in powers of momentum [37]. At present time, this framework is formulated only in a no-core shell model space, that is currently limited to light systems which can not exhibit most of collective exotic modes here discussed [38].

Recently the Hartree-Fock plus RPA based on correlated realistic NN interaction $V_{U C O M}$ [11] has been established and employed in description of collective low-amplitude motion in nuclei [39, 40] and it has further been extended toward SRPA [34]. However, exotic structure phenomena in nuclei away from the valley of stability still represent unsolved problem for theory frameworks based on low-momentum NN interactions $V_{\text {low }-k}$ and $V_{U C O M}$, as well as inter-nucleon forces derived within the $\chi$ EFT [13]. As pointed out in modeling the excitation spectra of light nuclei, e.g. ${ }^{10,11} \mathrm{~B},{ }^{12,13} \mathrm{C}$, based on no-core shell model with $\mathrm{NN}$ and NNN interactions derived within $\chi \mathrm{EFT}$, the inclusion of NNN force provides important contribution and improves the theory results in comparison with experiment [41. The crucial open problem that remains to be solved, is to provide quantitative and simultaneous description of nuclear ground state properties (e.g. binding energies, radii, nucleon separation energies, etc.) together with excitation energies and transition strengths of giant resonances and exotic modes of excitation. Within the existing theory frameworks, based on $V_{l o w-k}$ or $V_{U C O M}$, there are difficulties to reach simultaneous quantitative description of excitation energies of monopole, dipole and quadrupole giant resonances in stable nuclei, and fine structure properties such as low-energy excitations in nuclei away from the valley of stability still remain out of reach [39, 34]. Since the nuclear many body theory based on realistic NN interactions also necessitates inclusion of the NNN force, its implementation may improve the present status on description of collective modes of excitation and could open perspectives for studies of exotic modes in nuclei away from the valley of stability.

\section{Multipole excitations in neutron-rich nuclei}

In recent years the PDR has been at the center of theoretical research on exotic modes of excitation in nuclei with neutron excess [1], partly owing to profound progress in experimental studies, mainly based on photon scattering and Coulomb dissociation of 
radioactive ion beams, which resulted in valuable experimental data and opened new problems in understanding the nature of low-energy excitations in nuclei with isospin asymmetry. In the following, an overview is given for the critical questions about the nature of PDR and exotic multipole excitations, including both theoretical and experimental aspects that are currently not fully understood.

(i) Are the low-energy dipole states attributed to the PDR collective?

In contrast to giant resonances which are characterized by collective motion of nucleons, there are contradictory results on collectivity and nature of the PDR [1]. From the theory side, there is still no consensus whether the low-lying dipole strength could be attributed to a collective excitation mode. Whereas the relativistic quasiparticle random phase approximation (RQRPA), by its amplitudes and transition matrix elements, provides clear evidence about degree of collective nature of the PDR [1, 42], other self-consistent theory frameworks provide rather limited evidence supporting the notion of resonant structure of the PDR.

(ii) Where is the exact location of the PDR excitation energy?

Similar as in the case of giant resonances, the PDR centroid energy decreases with the neutron excess, resulting in characteristic crossing between the PDR excitation energy and the neutron separation energy for various isotope chains (e.g. Ni, Sn, and $\mathrm{Pb}$ ) [43]. For lower-mass isotopes the PDR is located below the neutron threshold, whereas in more neutron rich isotopes the PDR energy is higher than the neutron separation energy. Consequently, it is expected that there are important uncertainties in currently available data on low-energy transition strength in nuclei with isospin asymmetry where the PDR energy is close or above the neutron threshold. Although there is a considerable amount of successful $\left(\gamma, \gamma^{\prime}\right)$ studies (e.g. Refs. [19, 20, 21, 22, 23, 24, 25]), data on dipole excitations are available only below the neutron separation threshold, i.e. high-energy tail of the PDR may be missing.

(iii) Can one resolve the missing E1 strength in photon scattering experiments?

An important open problem in understanding the nature of PDR arises due to considerable amount of missing strength in $\left(\gamma, \gamma^{\prime}\right)$ measurements. The RQRPA calculations of the low-lying dipole strength along Sn isotope chain resulted in significantly larger amount of B(E1) transition strength than experimental values from photon scattering [1, 26]. Recent $\left(\gamma, \gamma^{\prime}\right)$ study confirmed that quite a considerable amount of low-energy dipole transition strength could not be observed with existing experimental techniques and methods [21], i.e. the missing strengths can vary from a few percent to factor of three, depending on the nucleus under consideration. Novel experimental facilities, e.g. the NEPTUN tagger facility [22], could provide information not only about dipole transitions below the neutron threshold, but also across and above, and could possibly recover significant part of the missing low-energy dipole strength.

(iv) Is there a connection between the PDR in stable and exotic nuclei?

An important open problem related to the PDR is resolving the connection between its nature in stable nuclei and those away from the valley of stability. This connection may not be smooth in approaching the neutron drip-line, where additional new structure 
phenomena may appear, e.g. di-neutron vibration modes [28]. Although a general trend of increasing pygmy dipole strength with neutron-proton asymmetry $\alpha=(N-Z) / A$ has been established [27], there are uncertainties in establishing systematic dependence on $\alpha$.

Most of available experimental data on the low-lying dipole excitations related to the PDR in nuclei with isospin asymmetry are rather close to the valley of stability. The pioneering experiment on the PDR in unstable nuclei, exploiting Coulomb dissociation of high-energy radioactive beams, provided crucial information about low-energy dipole spectra for ${ }^{130,132} \mathrm{Sn}$ [14]. However, due to the experimental techniques employed, the transition strength is provided only at energies above the neutron separation energy which is rather high, and some part of the PDR low-energy tail might be missing. Similar is in the case of low-energy transition strength obtained in the $\gamma$ decay from Coulomb excitation of ${ }^{68} \mathrm{Ni}[16]$.

(v) What is the macroscopic picture of nucleon vibrations in the PDR?

Studies probing the nature of PDR result in contradicting results. Whereas the RPA transition densities indicate that the PDR corresponds to the skin oscillation mode, where excess of weakly bound neutrons oscillate against the proton-neutron core [1], the studies based on quasiparticle phonon model showed that the PDR is characterized by vortical motion of nucleons [44]. On the other hand, relativistic RPA study of pronounced low-energy states obtained for isoscalar dipole and toroidal operators resulted in vortical motion in nucleon velocity fields as a genuine property that is inherent for the low-lying dipole states [45]. Therefore, it is not fully understood whether one could establish a connection between the vortical nature of low-lying dipole states and those of the PDR, i.e. if there is a link between the neutron skin and toroidal nuclear motion.

(vi) What is the nature of the isoscalar-isovector splitting of the PDR?

At present time, very little is known from the experimental side about the underlying structure of low-energy excitations, and available data mainly include excitation energies and B(E1) values for low-lying dipole states. Whereas transition densities have been measured in the past for giant resonances, due to experimental difficulties in the case of PDR these are not available, and alternative methods are needed to understand how neutrons and protons oscillate in the low-energy modes. An important study along these lines, based on $\left(\gamma, \gamma^{\prime}\right)$ and $\left(\alpha, \alpha^{\prime} \gamma\right)$ scattering, indicated that the structure of the low-energy excitations seems to be rather complex, i.e. the lower part appears more sensitive to the isocalar probe, whereas the higher part could be excited in both cases [46. The corresponding RQRPA study indicated that the more pronounced pygmy structure at lower energy is composed of predominantly isoscalar states with surface-peaked transition densities [42]. At somewhat higher energy the calculated E1 strength is primarily of isovector character, as expected for the low-energy tail of the giant dipole resonance. However, the double hump structure of the low-lying strength could not be reproduced by self-consistent model calculations, i.e. in the case of RQRPA this was possible only by introducing the experimental single-particle levels. 
This opens another important problem in the current implementations of the QRPA; mean field theory frameworks should be further developed in order to provide improved description of single-particle spectra for the relevant states around the Fermi level.

(vii) Are there other exotic multipole excitations in unstable nuclei?

Although at present time the most prominent exotic mode in neutron-rich nuclei is the PDR, one could expect that other exotic modes could appear in almost any multipolarity and isospin channel in nuclei away from the valley of stability. In particular, there is a whole set of open problems, related to discerning the conditions for existence of exotic monopole, quadrupole and octupole modes of excitation in nuclei with pronounced isospin asymmetry. Crucial question to be resolved is whether loosely bound nucleons, with wave functions spatially extended far beyond radii characteristic for stable isotopes, could coherently contribute to novel excitation modes of various multipolarities. In a recent study based on quasiparticle phonon model, quadrupole excitations in neutron-rich Sn isotopes have been explored above the first low-lying collective state and below the particle threshold [47]. It has been shown that the structure properties of these states are closely related to the neutron excess, indicating the existence of a skin oscillation quadrupole mode. This result has not been independently confirmed by self-consistent theory frameworks and it represents an open problem to be addressed.

Self-consistent models currently available to conduct studies along these lines, are usually formulated in the random phase approximation, which limits the field of research to low-amplitude motion only. Therefore, additional efforts are needed to develop new self-consistent theory frameworks based on modern effective interactions, which could describe vibrations in nuclei without imposing limitations on the vibration amplitude.

\section{Excitations in proton drip-line nuclei}

Nuclei close to the proton drip-line are characterized by unique structure properties due to the weakly bound proton orbitals and the states which are bound only because of the presence of the Coulomb barrier. The proton drip line is much closer to the line of stability than the neutron drip line. Bound nuclei with an excess of protons over neutrons can be found only in the region of light $\mathrm{Z} \leq 20$ and medium mass 20 $<\mathrm{Z} \leq 50$ elements. Due to the presence of the Coulomb barrier, nuclei close to the proton drip line generally do not exhibit a pronounced proton skin, except for very light elements. Recent studies of proton rich medium-mass nuclei, based on RQRPA and continuum RPA, showed that low-energy dipole states appear close to the proton drip line, which correspond to the proton PDR [48, 49]. The analysis of the proton and neutron transition densities showed that proton PDR states correspond to the oscillation of the proton excess against an approximately isospin-saturated core. The RQRPA amplitudes of the pygmy states are composed of many proton two-quasiparticle configurations, where dominant configurations include weakly bound proton orbitals and also the states which are bound only because of the presence of the Coulomb barrier. 
Apart from a limited number of studies related to dipole transitions, excitation modes in proton drip-line nuclei are at present time vastly unknown. These excitations are interesting not only as unique structure phenomena at the limits of stability, but also due to possible astrophysical relevance, e.g., in rp-process. Two-proton decay has been for the first time introduced by theory [50] and only recently it has been experimentally confirmed in the vicinity of the proton drip-line [51]. On the other hand, two-proton radiative capture, dominated by the E1 process, has been studied under conditions of stellar environment [52, 53]. However, the role of the proton PDR and its structure properties in two-proton capture rates still remains an open problem, and it has only been studied using three-body model for two-proton halo candidate ${ }^{17} \mathrm{Ne}$ [54]. Selfconsistent theoretical models based on modern effective interactions, which also include the role of the particle continuum, have not yet systematically been included in modeling these reactions.

\section{Charge-exchange modes and weak interaction rates}

Theoretical description of astrophysically relevant weak interaction of charged and neutral leptons with nuclei crucially depends on modeling reaction transitions from the nuclear initial to excited states. One of the major open problems in nuclear physics applications in astrophysics, is construction of fully self consistent microscopic theory framework based either on modern energy density functionals or realistic NN interactions supplemented by the NNN force, that could provide quantitative description of excitations contributing to weak interaction rates and allow extrapolations to unknown regions of nuclide map important for nucleosynthesis reactions, e.g. along the r-process path. In the evolution of supernova core collapse, nuclear reactions involving weak interaction play an essential role [55]. In particular, $\beta$-decay and electron capture in the mass region of iron nuclei create neutrinos and influence the number of electrons. Since neutrinos leave the star almost unhindered, in this way the stellar core is cooling and keeping its entropy low [56]. On the other hand, changing the number of electrons determines the pressure in the star during collapse, and could have direct impact on the dynamics of core collapse. Neutrino-nucleus reactions can also contribute to the dynamics of the collapse and the subsequent explosion phase [57] and their modeling necessitate self-consistent description of nuclear excitations induced by the neutrino interaction with nuclei.

Microscopic models for description of astrophysically relevant excitations are based on nuclear shell model or QRPA. At present time, weak-interaction cross sections for target nuclei beyond the $p f$-shell, and those including transitions of higher multipolarities, cannot be systematically evaluated with large-scale diagonalisation shell-model due to huge configuration spaces and the lack of a reliable effective interaction in other mass regions. Hybrid models have been introduced, which combine the shell-model Monte Carlo (SMMC) together with the RPA [58], in order to also provide spin-dipole and other excitations of higher multipolarities needed, e.g., for 
description of neutrino-nucleus cross sections and electron capture rates. In a recent application of the hybrid model in description of neutrino-nucleus reactions, the isobaric analogue state (IAS) and Gamow-Teller (GT) transition strengths are calculated in the shell-model with new Hamiltonian for $p f$ shell, while excitations of $J>1$ multipolarities are obtained from the RPA [59]. In modeling weak interaction rates, shell model can provide only IAS and GT excitations, whereas other excited states still remain out of the reach due to limitations in the configuration space. Obviously, development of novel theoretical frameworks is needed, that could in a systematic and consistent way provide all the necessary transitions throughout the nuclide map, including nuclei at the limits of stability. Although these objectives can be achieved starting from the self-consistent mean field theory frameworks supplemented with the (quasiparticle) RPA [60, 61, 62, 63], in this way important correlations inherent in the shell model are not taken into account.

At present time, the properties of charge-exchange excitations in exotic nuclei remain mainly unknown both from theoretical and experimental perspective. Similar as in the case of excitations without charge exchange, one could, at least in principle, expect that weakly bound nucleons could support formation of novel modes of excitations involving isospin flip. The evolution of the properties of basic excitation modes toward drip lines still remains unknown (e.g. spin-dipole excitations). Detailed knowledge about these and other charge-exchange excitations of multipolarities up to $\mathrm{J} \approx 6$ is crucial in description of weak interaction rates, especially at somewhat higher energies of incoming lepton projectile (e.g. electron, neutrino, etc.) [64]. An open problem to be resolved is to establish a novel theory framework for self-consistent description of the single particle properties, occupation probabilities and charge-exchange excitations in a systematic way throughout the nuclide map, by including higher order correlations going beyond the ph configurations inherent in RPA.

\section{Exotic modes of excitation in deformed nuclei}

Deformed nuclei represent a great challenge in the quest for novel modes of excitation and their description necessitates framework where deformation is explicitly taken into account. Only very recently, fully self-consistent theory frameworks for deformed nuclei have been established, based on quasiparticle random approximation with axial symmetry, formulated using Gogny effective force [65], as well as Skyrme [66] and covariant [67, 68] energy density functionals. The QRPA allows systematic description of various multipole transitions throughout the map of nuclides, including nuclei near the drip lines. As pointed out in Ref. [66], these approaches have practical drawback due to necessity to include a large two-quasiparticle cutoff which constrains the configuration space, and therefore computing times and memory storage are high. However, implementation of parallel codes [67] and on-going development of computing facilities will allow the configuration space to be considerably enlarged for the purposes in the quest for novel modes of excitation in deformed nuclei.

The evolution of the low-lying strength in deformed nuclei is determined by the 
interplay of isospin asymmetry and deformation; greater neutron excess increases the total low-lying strength while deformation results in spreading and hindering of the transition strength. In a model of a deformed nucleus in which protons and neutrons have been described as interacting rigid rotors with axial symmetry, magnetic-dipole collective state describing scissor-like rotational oscillations of protons against neutrons have been predicted thirty years ago [69]. The scissors mode has been a topic in numerous theoretical studies, and it has been experimentally observed in many nuclei by scattering of protons, electrons or photons [70, 71]. However, excitations in deformed nuclei away from the valley of stability are mainly unknown and present an open problem for future studies. In fact, only very recently the model calculations indicated that in neutron-rich ${ }^{154} \mathrm{Sm}$ a magnetic dipole state of unique structure properties appears at low excitation energy at $2.5 \mathrm{MeV}$ [72]. It has been shown that this peak corresponds to a novel exotic type of resonance with pronounced isoscalar character in which the neutron skin oscillates in a scissor like motion against the proton-neutron core. Apart from a few recent studies, excitations in exotic nuclei with deformation are at present time mainly unknown. These modes, both in neutron- and proton-rich nuclei, present a challenge not only for both relativistic and nonrelativistic energy density functionals, but also for models based on realistic NN and NNN interactions.

\section{Excitations in nuclei at finite temperature in stellar environment}

Nuclei at finite temperature are characterized by thermal population of single-particle states and modifications of nuclear structure properties, resulting in new features in excitation spectra. In general, description of open-shell nuclei necessitates a consistent treatment of pairing correlations together with finite temperature effects like, for instance, in the finite temperature HFB+QRPA framework 73. The phase transition from a superfluid to a normal state occurs at temperatures $T \approx 0.5-1 \mathrm{MeV}$ [74], whereas for temperatures above $T \approx 4 \mathrm{MeV}$ contributions from states in the continuum become large, and additional subtraction schemes have to be implemented to remove the contributions of the external nucleon gas [75].

Multipole responses in hot nuclei have been explored in a variety of theoretical frameworks, e.g. RPA based on schematic interactions [76], linear response theory [77], self-consistent Hartree-Fock plus RPA based on Skyrme functionals [78, 79], and covariant energy density functionals [80]. The decay of hot nuclei and thermal damping of giant resonances have also been studied in great detail [81, 82]. Whereas the main giant resonance peaks remain weakly affected at finite temperatures up to $\mathrm{T}=3 \mathrm{MeV}$, long low-energy tails have been obtained.

In view of all the previous sections in this article, crucial topic that is currently not understood is the question how various exotic modes of excitation evolve with increased temperature, in particular under conditions of the stellar environment where these modes could play significant role in astrophysical processes (cf. Sec. 6). Since the major effect due to finite temperature is expected in the low-energy part of the excitation spectra, 
one could anticipate considerable modifications in the properties of exotic modes of excitation or novel transition strength may appear [80].

Particular challenge is self-consistent description of structure properties of excitation modes in nuclei at the limits of stability at temperatures in stellar conditions. In addition to weak binding of outermost nucleons, finite temperature further redistributes the occupation numbers of single-particle states, resulting in additional weakening of the nuclear binding and modifications in excitation spectra. At temperatures below the phase transition from a superfluid to normal state $(T<0.5-1$ $\mathrm{MeV}$ ), the structure properties will depend on the interplay between the effects of the pairing correlations and finite temperature, resulting in partial occupations of relevant single-particle states around the Fermi level, which contribute to the excitation configuration space.

While the finite temperature effects on giant resonances are rather moderate, it is not understood to what extent finite temperature would support the existence of exotic modes, or what are conditions for these modes to become suppressed. Whereas some of these issues could be addressed by self-consistent theory frameworks based on modern energy density functionals, the knowledge from the experimental side is mainly limited to giant resonances [83, 84, 85], and considerable efforts are necessary to provide some information about the structure properties of weakly bound nuclei at finite temperature. At present time, even very basic information needed for description of weak interaction rates is not available from experiment, namely the evolution of $\beta^{+}$Gamow-Teller transition strength with temperatures in the range characteristic for presupernova conditions of stars (e.g. $\mathrm{T}=0-2 \mathrm{MeV}$ ). Although $G T^{ \pm}$transitions dominate $\beta$-decays and electron captures under supernova core-collapse conditions [55], forbidden transitions could also result in non-negligible contributions in astrophysical applications, as shown for instance in the case of $\beta$-decays of nuclei near the r-process path [86]. Therefore, forbidden transitions contributing to the weak interaction rates should be revised within self-conistent theory frameworks at finite temperature, as well as their role in astrophysical applications.

\section{Conclusion}

At present time, both theoretical and experimental studies of exotic modes of excitation are to a large extent focused on discerning the nature of pygmy dipole resonances. As discussed in Sec. 3, due to considerable progress in theoretical and experimental research within recent years, several questions are opened on the underlying structure properties of the PDR, including the issues of collectivity, location of the PDR with respect to neutron threshold energy, the missing strength from photon scattering experiments, connection between the PDR in stable nuclei and those at the frontiers of stability, macroscopic picture of nuclear vibrations, and isoscalar-isovector splitting of the PDR. On the other side, nuclei at extreme isospin and finite temperature, as well as deformed nuclei with isospin asymmetry, open perspectives for a whole new 
class of monopole, dipole, quadrupole and octupole excitation modes, as well as exotic charge-exchange vibrations. Since nuclei at finite temperature and away from the valley of stability participate in astrophysically relevant reactions, new knowledge on exotic nuclear modes of excitation may result in important consequences in understanding the processes in stellar environment. In the quest for novel exotic modes of excitation, development of theoretical frameworks plays a central role. Reliable microscopic description of excitations necessitates fully self-consistent theory, able to include the effects of the particle continuum and couplings to complex configurations, in order to provide description of both escape and spreading widths. These could be formulated by using modern energy density functionals constrained by the strong interaction physics of QCD and/or by the phenomenological input. On the other side, exotic modes of excitation also represent a great challenge and valuable test for theory frameworks based on realistic NN interactions, including low-momentum NN interactions, $V_{\text {low }-k}$ and $V_{U C O M}$, as well as chiral NN interactions, supplemented by three body forces.

\section{ACKNOWLEDGMENTS}

This work was supported by the Unity through Knowledge Fund (UKF Grant No. 17/08) and Ministry of Science, Education and Sports of the Republic of Croatia (project No. 1191005-1010) and Croatian National Foundation for Science.

\section{References}

[1] Paar N, Vretenar D, Khan E, and Colò G 2007 Rep. Prog. Phys. 70691

[2] Bender M, Heenen PH, Reinhard PG 2003 Rev. Mod. Phys. 75121

[3] Dobaczewski J, Stoitsov MV, Nazarewicz W, Reinhard PG 2007 Phys. Rev. C 76054315

[4] Carlsson BG, Dobaczewski J, Kortelainen M 2008 Phys. Rev. C 78044326

[5] Kortelainen M, Dobaczewski J, Mizuyama K, Toivanen J 2008 Phys. Rev. C 77064307

[6] Colò G, Bortignon PF 2008 Eur. Phys. J. Special Topics 156229

[7] Finelli P, Kaiser N, Vretenar D, Weise W 2006 Nucl. Phys. A 7701

[8] Nikšić T, Vretenar D, Ring P 2008 Phys. Rev. C $\mathbf{7 8} 034318$

[9] Ring P, Litvinova EV 2009 Phys. Atom. Nucl. 721285

[10] Bogner SK, Kuo TTS, Schwenk A 2003 Phys. Rep. 3861

[11] Feldmeier H, Neff T, Roth R, and Schnack J 1998 Nucl. Phys. A 63261

[12] Bogner SK, Furnstahl RJ, Schwenk A 2009 arXiv:0912.3688

[13] Epelbaum E, Hammer HW, Meissner UG 2009 Rev. Mod. Phys. 811773

[14] Adrich P et al. 2005 Phys. Rev. Lett 95132501

[15] Aumann T 2005 Eur. Phys. J. A 26441

[16] Wieland O 2009 Phys. Rev. Lett 10209250

[17] Khan E, Blumenfeld Y, Van Giai N, et al. 2000 Phys. Lett. B 49045

[18] Burda O, Botha N, Carter J, et al. 2007 Phys. Rev. Lett 99092503

[19] Hartmann T, Enders J, Mohr P, et al. 2000 Phys. Rev. Lett 85274

[20] Zilges A, Volz S, Babilon M, et al. 2002 Phys. Lett. B $\mathbf{5 4 2} 43$

[21] Savran D et al. 2008 Phys. Rev. Lett. 100232501

[22] Savran D et al. 2009 Nucl. Instr. Methods, Phys. Res. Sect. A, in press

[23] Schwengner R, Rusev G, Benouaret N, et al. 2007 Phys. Rev. C 76034321

[24] Wagner A, Beyer R, Erhard M, et al. 2008 J. Phys. G 35014036

[25] Rusev G, Schwengner R, Beyer R, et al. 2009 Phys. Rev. C 79061302 
[26] Özel B, Enders J, von Neumann-Cosel P, Poltoratska I, Richter A, Savran D, Volz S, Zilges A 2007 Nucl. Phys. A $\mathbf{7 8 8} 385 \mathrm{c}$

[27] Klimkiewicz A et al. 2007 Phys. Rev. C 76 051603(R)

[28] Matsuo M, Mizuyama K and Serizawa Y 2005 Phys. Rev. C 71064326

[29] Khan E, Sandulescu N, Grasso M, Van Giai N 2002 Phys. Rev. C 66024309

[30] Mizuyama K, Matsuo M, Serizawa Y 2009 Phys. Rev. C 79024313

[31] Nakada H, Mizuyama K, Yamagami M, and Matsuo M 2009 Nucl. Phys. A 828283

[32] Litvinova E, Ring P, Tselyaev V 2008 Phys. Rev. C 78014312

[33] Litvinova E, Ring P, Tselyaev V, Langanke K 2009 Phys. Rev. C 79054312

[34] Papakonstantinou P and Roth R 2009 Phys. Lett. B 671356

[35] Gambacurta, Grasso M, and Catara F 2010 arXiv:1002.3563

[36] Finelli P, Kaiser N, Vretenar D, Weise W 2007 Nucl. Phys. A 79157

[37] Bedaque PF, van Kolck U 2002 Ann. Rev. Nucl. Part. Sci. 52339

[38] Stetcu I, Rotureau J, Barrett BR, van Kolck U 2009 arXiv:0912.3015

[39] Paar N, Papakonstantinou P, Roth R, and Hergert H 2006 Int. J. Mod. Phys. 15346

[40] Paar N, Papakonstantinou P, Hergert H, Roth R 2006 Phys. Rev. C 74014318

[41] Navrátil P, Gueorguiev VG, Vary JP, Ormand WE, Nogga A, Quaglioni S 2008 Few Body Syst. 43129

[42] Paar N, Niu YF, Vretenar D, and Meng J 2009 Phys. Rev. Lett 103032502

[43] Paar N, Nikšić T, Vretenar D, Ring P 2005 Phys. Lett. B 606288

[44] Ryezayeva N et al. 2002 Phys. Rev. Lett. 89272502

[45] Vretenar D, Paar N, Ring P, Nikšić T 2002 Phys. Rev. C 65021301

[46] Savran D et al. 2006 Phys. Rev. Lett 97172502

[47] Tsoneva N and Lenske H 2009 arXiv:0910.3487

[48] Paar N, Vretenar D, and Ring P 2005 Phys. Rev. Lett. 94182501

[49] Paar N, Papakonstantinou P, Ponomarev V Yu, Wambach J 2005 Phys. Lett. B 624195

[50] Goldansky VI 1969 Nucl. Phys. 19482

[51] Pfützner M et al. 2002 Eur. Phys. J. A 14279

[52] Görres J, Wiescher M, Thielemann FK 1995 Phys. Rev. C 51392

[53] Grigorenko LV and Zhukov MV 2005 Phys. Rev. C 72015803

[54] Grigorenko LV, Langanke K, Shul'gina NB, Zhukov MV 2006 Phys. Lett. B 641254

[55] Janka H Th, Langanke K, Marek A, Martínez-Pinedo G, Müller 2007 Phys. Rep 44238

[56] Bethe HA, Brown GE, Applegate J, Lattimer JM 1979 Nucl. Phys. A 324487

[57] Haxton WC 1988 Phys. Rev. Lett 601999

[58] Langanke K, Kolbe E, and Dean DJ 2001 Phys. Rev. C 63 032801(R)

[59] Suzuki T, Honma M, Higashiyama K, Yoshida T, Kajino T, Otsuka T, Umeda H, and Nomoto K 2009 Phys. Rev. C 79 061603(R)

[60] Fracasso S, Colò G 2005 Phys. Rev. C 72064310

[61] Paar N, Niksic T, Vretenar D, Ring P 2004 Phys. Rev. C 69054303

[62] Liang HZ, Van Giai NY, Meng J 2008 Phys. Rev. Lett 101122502

[63] Liang HZ, Van Giai N, Meng J 2009 Phys. Rev. C 79064316

[64] Paar N, Vretenar D, Marketin T, Ring P 2008 Phys. Rev. C 77, 024608

[65] Peru S, Goutte H, and Berger JF 2007 Nucl. Phys. A 788 44c

[66] Yoshida K and Van Giai N 2008 Phys. Rev. C 78064316

[67] Peña Arteaga D and Ring P 2008 Phys. Rev. C 77034317

[68] Peña Arteaga D, Khan E, and Ring P 2009 Phys. Rev. C 79034311

[69] Lo Iudice N and Palumbo F 1978 Phys. Rev. Lett. 411532

[70] Bohle D, Richter A, Steffen W, Dieperink AEL, Iudice NL, Palumbo F, Scholten O 1984 Phys. Lett. B 13727

[71] Kneissl U et al. 1996 Progr. Part. Nucl. Phys. 37349

[72] Peña Arteaga D and Ring P 2009 arXiv:0912.0908 
[73] Sommermann HM 1983 Ann. Phys. 151163

[74] Khan E, Van Giai N, and Grasso M 2004 Nucl. Phys. A 731311

[75] Bonche P, Levit S, and Vautherin D 1984 Nucl. Phys. A 427278

[76] Civitarese O, Broglia RA, and Dasso CH 1984 Ann. Phys. 156142

[77] Faber ME, Egido JL, and Ring P 1983 Phys. Lett. B 1275

[78] Sagawa H and Bertsch GF 1984 Phys. Lett. B 146138

[79] Paar N, Colò G, Khan E, and Vretenar D 2009 Phys. Rev. C 80055801

[80] Niu YF, Paar N, Vretenar D, and Meng J 2009 Phys. Lett. B 681315

[81] Gallardo M, Diebel M, Dossing T, and Broglia RA 1985 Nucl. Phys. A 443415

[82] Bortignon PF, Broglia RA, Bertsch GF, and Pacheco J 1986 Nucl. Phys. A 460149

[83] Bracco A et al. 1989 Phys. Rev. Lett. 622080

[84] Baumann T et al. 1998 Nucl. Phys. A 635428

[85] Santonocito D and Blumenfeld Y 2006 Eur. Phys. J. A 30183

[86] Borzov IN 2003 Phys. Rev. C 67025802 